\title{
Influence of the Stimulation Current on the Differences Between Cell and Tissue Electrophysiological Simulations
}

\author{
V Monasterio ${ }^{1}$, E Pueyo ${ }^{2,3}$, J F Rodríguez-Matas ${ }^{4}$ J Carro $^{1}$ \\ ${ }^{1}$ Universidad San Jorge, Villanueva de Gállego, España \\ ${ }^{2}$ Aragón Institute for Engineering Research, University of Zaragoza, IIS Aragón, Spain \\ ${ }^{3}$ CIBER in Bioengineering, Biomaterials and Nanomedicine (CIBER-BBN), Spain \\ ${ }^{4}$ LaBS, Department of Chemistry, Materials and Chemical Engineering "Giulio Natta", Politecnico \\ di Milano, Italy
}

\begin{abstract}
This study compares stimulation waveforms for singlecell simulations. The alternatives include monophasic and biphasic current pulses, and a new waveform that resembles the transmembrane current responsible for conduction in tissue. Results indicate that the new stimulation produces the lowest mismatch between action potential markers simulated in cell and in tissue. In comparison with the monophasic stimulation, the new stimulation reduced cell-fiber differences by $99 \%$ for triangulation, by $95 \%$ for maximum transmembrane voltage, and by $76 \%$ for the maximum voltage slope. In conclusion, the new stimulation waveform could help to improve the trustworthiness of single-cell simulations in studies involving tissuederived markers.
\end{abstract}

\section{Introduction}

Cardiac computational models are a valuable tool for improving our understanding of cardiac electrical activity. In particular, action potential (AP) models of cardiac myocytes can be used to understand cellular electrical function under physiological conditions or in response to disease- or drug-induced alterations.

In silico models are generally validated by comparing simulated results with available experimental data. Experiments, however, exhibit a high degree of variability, both between cells and between subjects, while most current cell models only reproduce an AP meant to represent the "average" over a population. Rather than considering just a single AP model, a current trend in research is to create a population of models (PoM), that is, an ensemble of models covering a range of physiological characteristics such that they are able to represent the experimental electrophysiological variability. Computational simulations are run based on the created ensemble of models and the obtained results are compared to experimental observations. Candidate models whose results are not consistent with experiments are discarded. The remaining models constitute an experimentally-calibrated PoM [1]. During calibration, it is crucial that simulations mimic experimental conditions as closely as possible, including stimulation conditions.

Common electrophysiological markers for model calibration include AP duration (APD), maximum transmembrane potential or AP triangulation, among others. Due to the sensitivity of some ionic channels to cell isolation procedures, the reference experimental values for some of those markers are commonly obtained from cardiac tissue preparations. In such cases, although intactness of ion channels is preserved, the resulting characterization of electrophysiological properties may be strongly influenced by intercellular communication. Not accounting for intercellular coupling effects may inadvertently introduce a source of error when comparing simulations with experimental observations. Specifically, some studies in the literature [2] have pointed out that, for some AP-derived markers, there is not a close correspondence between values measured in cell and tissue. Those markers must be used with caution when developing or calibrating singlecell AP models if a comparison with experimental tissue data is going to be considered.

The cell-tissue mismatch could be avoided by simulating the behavior of cardiac tissue for calibration, but such approach would excessively increase the computational cost, since PoMs contain thousands of model variations. More feasible alternatives should try to incorporate, as far as possible, the effects of inter-cellular communication into single-cell simulations. In the literature, a biphasic stimulation has been proposed to imitate cell excitation during AP conduction [3]. The effect of such stimulation on the cell-tissue mismatch has not been studied yet.

In this work we analyze four stimulation waveforms ap- 
plied onto single-cell AP simulations and quantify the mismatch between AP markers measured in simulated cells and tissues. The waveforms include the usual monophasic and biphasic (symmetric and asymmetric) pulses as well as a new waveform that resembles the transmembrane current associated with AP conduction in tissue.

\section{Methods}

\subsection{Computational simulations}

Single-cell and fiber simulations were carried out using the ten Tusscher-Panfilov (TP06) human ventricular AP model as a basis [4].

For tissue simulations, we created a homogeneous 3cm-long fiber composed of epicardial cells. Electrical propagation across the cardiac tissue was modeled with a monodomain reaction-diffusion equation. Discretization was performed with a space step of $0.1 \mathrm{~mm}$ and a time step of $0.01 \mathrm{~ms}$. The value of the conductivity was set to obtain a conduction velocity close to $65 \mathrm{~cm} / \mathrm{s}$ under control conditions [5]. The system of equations was solved using the conjugate gradient method. The fiber was stimulated with a monophasic current pulse with an amplitude of twice the diastolic threshold, defined as the minimum amplitude required to propagate five APs, and a duration of $1 \mathrm{~ms}$. The simulation software ELVIRA was used for the computations [6].

For single-cell simulations, the system of ordinary differential equations was solved by using forward Euler integration with a time step of $0.01 \mathrm{~ms}$. We compared four waveforms for the stimulus current:

a) Monophasic pulse $(\mathrm{M})$ with a duration $(\Delta t)$ of $1 \mathrm{~ms}$ and an amplitude of $A \mathrm{pA} / \mathrm{pF}$.

b) Biphasic asymmetric pulse (BA), defined as in [3]:

$$
I^{B A}(t)= \begin{cases}A, & 0 \leq t \leq \Delta t \\ \frac{-A \Delta t}{T-\Delta t}, & \Delta t<t<T\end{cases}
$$

where $T$ is the pacing cycle length.

c) Biphasic symmetric pulse (BS), defined as:

$$
I^{B S}(t)= \begin{cases}A, & 0 \leq t \leq \Delta t \\ -A, & \Delta t<t \leq 2 \Delta t\end{cases}
$$

d) Adjusted waveform (A), defined as:

$$
I^{A}(t)= \begin{cases}A\left(\frac{\gamma-t}{\alpha}\right)^{\beta-1} e^{-\left(\frac{\gamma-t}{\alpha}\right)^{\beta}}, & \gamma-\Delta t<t \leq \gamma \\ -A\left(\frac{t-\gamma}{\alpha}\right)^{\beta-1} e^{-\left(\frac{t-\gamma}{\alpha}\right)^{\beta}}, & \gamma<t<\gamma+\Delta t\end{cases}
$$

where $\alpha=0.27, \beta=1.37$ and $\gamma=1.5$ are the scale, shape and location parameters of an inverted and timeshifted Weibull distribution. These values were obtained by adjusting the positive phase of the wave to the positive phase of a reference waveform extracted from the fiber control simulation (Fig. 1). The reference stimulation was computed as an estimation of the transmembrane current of the central cell in the fiber, that is,

$$
\hat{I}_{m}=-\left(C \frac{d V}{d t}+I_{i o n}\right),
$$

where $C$ is the capacitance per unit area of membrane (1 $\left.\mu F / \mathrm{cm}^{2}\right), V$ is the transmembrane potential and $I_{i o n}$ represents the sum of ionic currents in the model.

For waveforms M, BS and BA, $A$ was set to twice the diastolic threshold, and $\Delta t$ was set to $1 \mathrm{~ms}$. For the adjusted waveform, $A$ was set to $235.71 \mathrm{pA} / \mathrm{pF}$ and $\Delta t$ was set to $1.5 \mathrm{~ms}$ in order to match the reference waveform.

\subsection{Steady-state AP markers}

The AP markers proposed in [2] were computed from fiber and single-cell simulations. In particular, we computed the APD at different percentages of repolarization (90\%, $75 \%, 50 \%$ and $25 \%)$, AP triangulation as $A P D_{90}-$ $A P D_{50}$, the maximum and minimum transmembrane potential $\left(V_{\max }\right.$ and $\left.V_{\min }\right)$ and the maximum and minimum AP slope $\left(d V / d t_{\max }\right.$ and $\left.d V / d t_{\min }\right)$.

Control conditions were simulated by stimulating fiber and single-cell models for 100 cycles at $1 \mathrm{~Hz}$. AP markers were computed from the last AP. Single-cell and fiber results were compared by computing the relative difference between each marker $j$ obtained in single-cell simulations $\left(M_{j}^{c}\right)$ and in fiber simulations $\left(M_{j}^{f}\right)$ as

$$
E(\%)=\frac{M_{j}^{c}-M_{j}^{f}}{M_{j}^{f}} \cdot 100 .
$$

The individual contribution of each major ionic conductance to AP markers was assessed next. Each ionic conductance was varied by $\pm 15 \%$ and $\pm 30 \%$ and its role

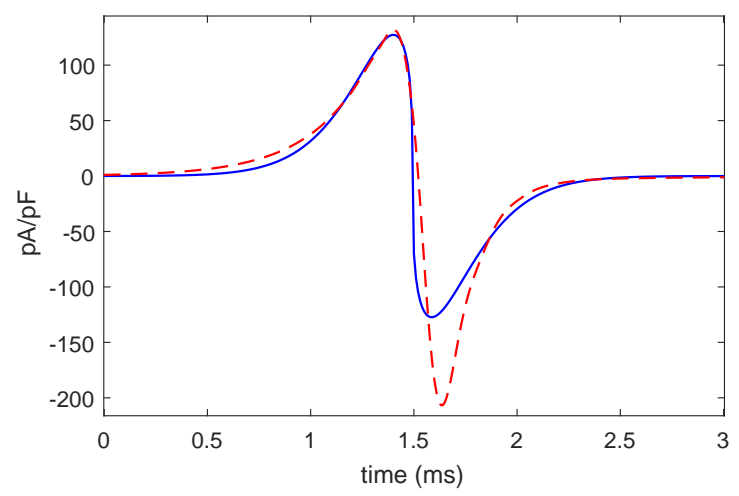

Figure 1. Dashed line: estimated transmembrane current $\hat{I}_{m}$. Solid line: adjusted waveform $I^{A}$. 
in determining each physiological AP-derived marker was evaluated by using a first order response surface model as proposed in [2].

\subsection{Response to stimulation frequency}

Five-minute simulations in single-cell and fiber were performed at a range of stimulation frequencies, ranging from $1 \mathrm{~Hz}$ to $4 \mathrm{~Hz}$. For each of those frequencies, the values of $A P D_{90}$ and intracellular potassium concentration, $\left[K^{+}\right]_{i}$, were assessed from the last simulated beat.

\section{Results}

At $1 \mathrm{~Hz}$ pacing (control), stimulation A produced the smallest difference between cell and fiber (Table 1) for all markers except for $A P D_{25}$. As compared to the monophasic stimulation (M), stimulation A produced a substantial reduction in the differences between cell and fiber for triangulation, $V_{\max }, d V / d t_{\max }$ and $d V / d t_{\min }$.

Regarding ionic contributors, the conductances of $I_{N a}$ and $I_{C a L}$ currents played different roles in fiber and cell simulations (Fig. 2). The impact of those two conductances on most electrophysiological AP markers was very similar when evaluated in fiber and in single cell stimulated with the A waveform. Of all analyzed markers, the most notable differences were found for AP triangulation, where stimulation M, BA and BS overestimated the contribution of the $I_{N a}$ current conductance and underestimated the influence of the $I_{\mathrm{CaL}}$ current conductance. Stimulation A produced the best match to fiber results for this marker. Stimulation A also improved the cell-fiber match in $V_{\max }$ and $d V / d t_{\min }$ markers, although in those cases additional contributors were found in cell but not in fiber ( $I_{t o}$ for $V_{\max } ; I_{K s}$ and $I_{b C a}$ for $d V / d t_{\min }$ ).

The values of $A P D_{90}$ and $\left[K^{+}\right]_{i}$ varied with the stimulation frequency in a similar fashion for fiber, BA, BS and A simulations (Fig. 3). Stimulation M led to overestimation of $\left[K^{+}\right]_{i}$ with respect to fiber. In Fig. 3, curves are shown up to the cycle length that matched the refractory period of the cells, which led to AP generation failure.

Table 1. Relative differences $\mathrm{E}(\%)$ between markers simulated in cell (with stimulation M, BA, BS and A) vs. fiber.

\begin{tabular}{lcccc}
\hline \hline Stimulation & M & BA & BS & A \\
\hline$A P D_{90}(\mathrm{~ms})$ & 0.1 & -1.5 & 0.0 & 0.0 \\
$A P D_{75}(\mathrm{~ms})$ & 0.0 & -1.7 & -0.3 & 0.0 \\
$A P D_{50}(\mathrm{~ms})$ & -1.4 & -3.1 & -2.4 & -0.1 \\
$A P D_{25}(\mathrm{~ms})$ & -10.9 & -12.7 & -0.3 & -0.5 \\
Trian. $(\mathrm{ms})$ & 18.6 & 16.8 & 28.4 & 0.9 \\
$V_{\max }(\mathrm{mV})$ & 51.7 & 52.6 & 72.4 & 2.4 \\
$V_{\min }(\mathrm{mV})$ & 0.0 & 0.3 & 0.0 & 0.0 \\
$d V / d t_{\max }(\mathrm{V} / \mathrm{s})$ & 65.7 & 67.2 & 66.5 & 15.8 \\
$d V / d t_{\min }(\mathrm{V} / \mathrm{s})$ & 168.8 & 173.9 & 1883.7 & 10.6 \\
\hline
\end{tabular}

\section{Discussion and conclusions}

This work presents a new model for the stimulus current in single cells that allows reproducing tissue electrophysiological characteristics. The model is based on the adjustment to a reference waveform extracted from simulations conducted in a fiber of cells. The resulting waveform mimics the source-sink behavior associated with AP propagation in tissue and is consistent with experimental observations [7]. In single cells stimulated with the adjusted waveform, our set of evaluated AP markers were found to present values similar to those measured in fiber. The adjusted stimulation also improved the match between the major ionic contributors to AP markers in single-cell and fiber simulations. This was not the case for the other tested stimulation waveforms. Consequently, this new stimulation can be a convenient alternative to the usual monophasic stimulation when a population of models needs to be calibrated with experimental data measured in tissue preparations, specially in those cases where AP triangulation, maximum voltage or voltage slopes are selected as markers for evaluation.

Regarding frequency behavior, our results are consistent with the literature [3]. In the TP06 cell model, stimulation is modeled as a $K^{+}$current. At high stimulation frequencies, monophasic stimulation pulses produce a remarkable accumulation of $\left[\mathrm{K}^{+}\right]_{i}$, which in turn alters $\left[\mathrm{Na}^{+}\right]_{i}$. BA stimulation was proposed to alleviate this effect [3] by including a negative phase to cancel out the total charge over one beat. The adjusted stimulation proposed in this work also prevents $\left[\mathrm{K}^{+}\right]_{i}$ overload and leads to AP generation failure at a frequency $(2.9 \mathrm{~Hz}$, Fig. 3) closer to that in fiber $(3.1 \mathrm{~Hz})$ than stimulation BA $(3.7 \mathrm{~Hz})$.

This work represents a first step towards an improved stimulation protocol for single-cell simulations. Further work could investigate variations in the adjusted stimulation waveform depending on additional factors, including the possibility to adjust it for each AP model. Nevertheless, given the high inter-cell and inter-subject variability in experimental AP properties, it is necessary to ponder to what extent a better cell-fiber match would justify the computational cost of specifically adjusting the stimulation waveform to each simulated condition. Our results indicate that the basic adjustment presented in this study could be enough to decrease the mismatch between cell and fiber and could ultimately help to improve the trustworthiness of PoM studies without adding computational cost.

\section{Acknowledgments}

This work was supported by projects DPI2016-75458-R (MINECO) and MULTITOOLS2HEART-ISCIII, by Gobierno de Aragón (Reference Group BSICoS T39_17R) cofunded by FEDER 2014-2020 "Building Europe from Aragon", and by European Research Council (ERC) through project ERC-2014-StG 638284. 


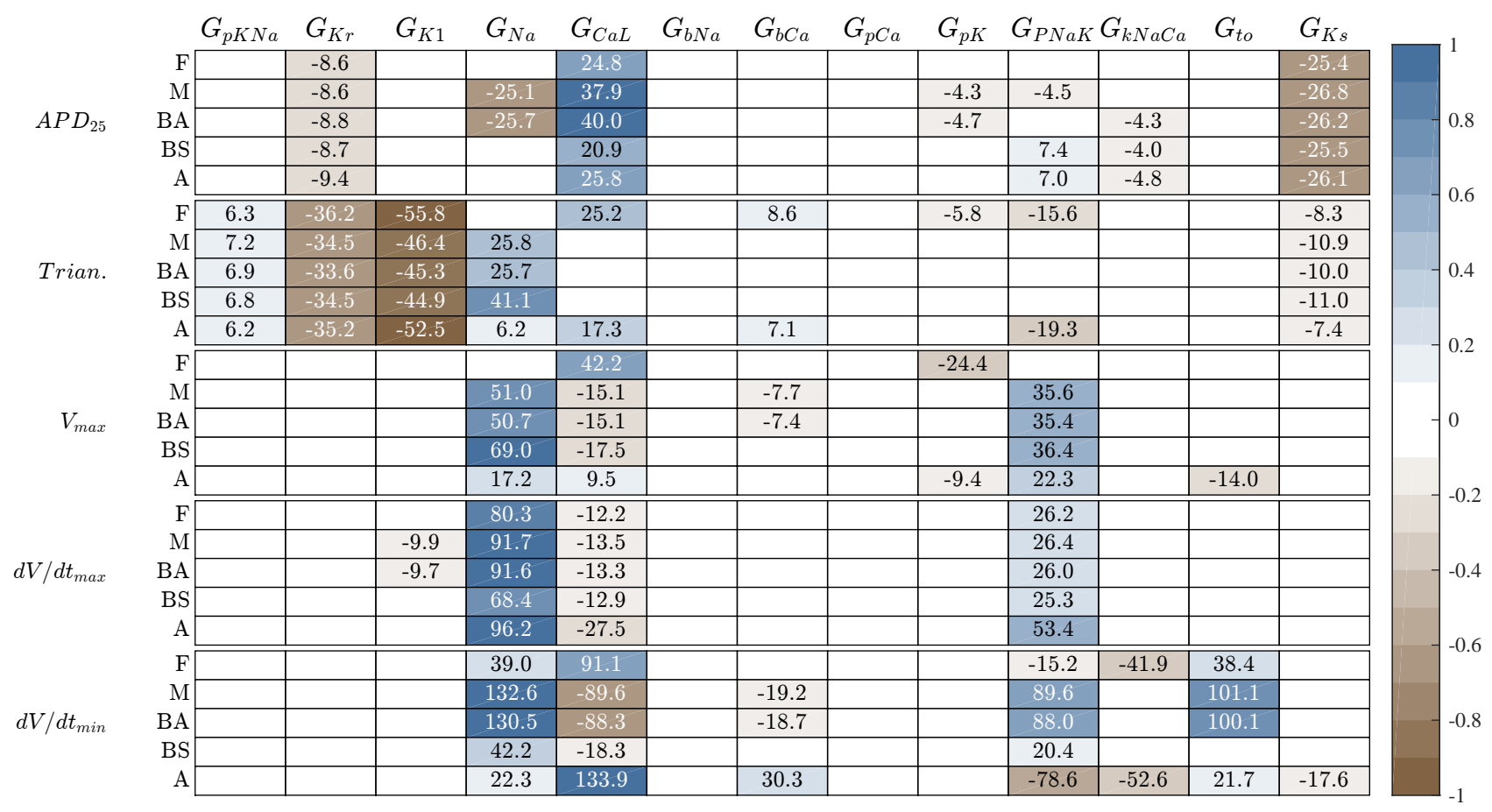

Figure 2. Contribution of ionic conductances to AP markers simulated in fiber (F) and cell (stimulation S, BA, BS and A). Color indicates the correlation between changes in a conductance and changes in a marker. Percentages in boxes indicate the contribution of changes in a conductance to changes in a marker.


Figure 3. Percentage of the initial value of $A P D_{90}$ and $\left[K^{+}\right]_{i}$ vs. stimulation frequency.

\section{References}

[1] Muszkiewicz A, Britton OJ, Gemmell P, Passini E, Sánchez C, Zhou X, Carusi A, Quinn TA, Burrage K, Bueno-Orovio A, Rodriguez B. Variability in cardiac electrophysiology: Using experimentally-calibrated populations of models to move beyond the single virtual physiological human paradigm. Prog Biophys Mol Biol 2016;120(1-3):115-127.

[2] Carro J, Rodríguez-Matas JF, Monasterio V, Pueyo E. Limitations in electrophysiological model development and validation caused by differences between simulations and experimental protocols. Prog Biophys Mol Biol 2017;129:53-64.

[3] Livshitz L, Rudy Y. Uniqueness and stability of action potential models during rest, pacing, and conduction using problem-solving environment. Biophys J 2009;97(5):12651276.

[4] ten Tusscher KHWJ, Panfilov Va. Alternans and spiral breakup in a human ventricular tissue model. Am J Physiol Heart Circ Physiol 2006;291(3):H1088-H1100.

[5] Taggart P, Sutton PM, Opthof T, Coronel R, Trimlett R, Pugsley W, Kallis P. Inhomogeneous transmural conduction during early ischaemia in patients with coronary artery disease. J Mol Cell Cardiol 4 2000;32(4):621-30.

[6] Heidenreich Ea, Ferrero JM, Doblaré M, Rodríguez JF. Adaptive macro finite elements for the numerical solution of monodomain equations in cardiac electrophysiology. Ann Biomed Eng 2010;38(7):2331-2345.

[7] Gray RA, Mashburn DN, Sidorov VY, Wikswo JP. Quantification of transmembrane currents during action potential propagation in the heart. Biophys J 2013;104(1):268-278.

Address for correspondence:

Violeta Monasterio (vmonasterio@usj.es)

Universidad San Jorge, 50830 Villanueva de Gállego, Spain 